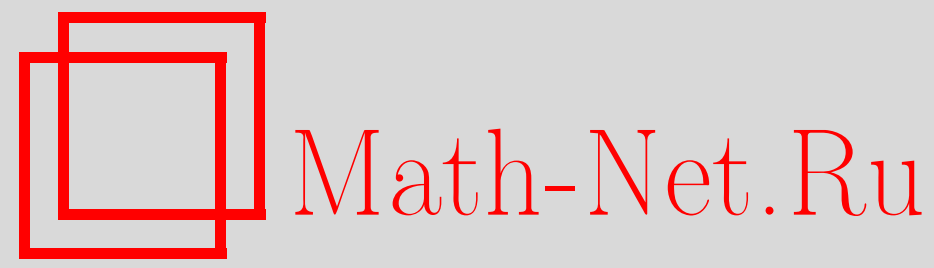

О. А. Козлитин, Вероятностные линейные соотношения в двоичных рекуррентных последовательностях, Матем. вопр. криптогр., 2017, том 8, выпуск 3, 57-84

DOI: https://doi.org/10.4213/mvk232

Использование Общероссийского математического портала Math-Net.Ru подразумевает, что вы прочитали и согласны с пользовательским соглашением

http://www.mathnet.ru/rus/agreement

Параметры загрузки:

IP : 18.207 .199 .55

26 апреля 2023 г., 16:03:36 
МАТЕМАТИЧЕСКИЕ ВОПРОСЫ КРИПТОГРАФИИ

2017 T. 8 № 3 C. $57-84$

УДК 519.12+519.719.2

\title{
Вероятностные линейные соотношения в двоичных рекуррентных последовательностях
}

\author{
О. А. Козлитин
}

ООО «Центр сертификационных исследований», Москва

Получено 30.V.2016

Статья посвящается памяти Александра Александровича Нечаева

\begin{abstract}
Аннотация. Рассматриваются алгебраические методы поиска вероятностных линейных соотношений, выполняющихся на отрезке двоичной линейной рекуррентной последовательности. Оценена трудоемкость предложенных алгоритмов.
\end{abstract}

Ключевые слова: вероятностное линейное соотношение, вероятностный характеристический многочлен, коэффициент статистической структуры

Probabilistic linear relations in binary recurring sequences

O. A. Kozlitin

Certification Research Center, LLC, Moscow

Abstract. We consider algebraic methods of searching for probabilistic linear relations on the segment of a binary linear recurring sequence. The complexity of suggested algorithms is estimated.

Keywords: probabilistic linear relation, probabilistic characteristic polynomial, coefficient of statistical structure

Citation: Mathematical Aspects of Cryptography, 2017, v. 8, № 3, pp. 57-84 (Russian)

(c) Академия криптографии Российской Федерации, 2017 г. 


\section{1. Введение}

Один из методов идентификации выходной последовательности двоичного автономного регистра сдвига заключается в следующем: для данной линейной комбинации $x_{i_{1}} \oplus x_{i_{2}} \oplus \cdots \oplus x_{i_{k}}$ знаков материала вычисляется ее преобладание. Если модуль преобладания превышает заданную границу $C>0$, то критерий принимает гипотезу о том, что данный отрезок является выходом автономного регистра сдвига. В противном случае принимается гипотеза о том, что данный отрезок получен по случайной равновероятной схеме.

Следует отметить, что указанный подход к идентификации линейных рекуррентных последовательностей в определенном смысле устойчив к искажениям. Действительно, неискаженную рекурренту можно идентифицировать при помощи алгоритма Берлекемпа-Месси (см., например, [6]). Однако на практике имеющийся участок рекурренты, как правило, искажен. Если количество искаженных битов таково, что модуль преобладания линейной комбинации $x_{i_{1}} \oplus x_{i_{2}} \oplus \cdots \oplus x_{i_{k}}$ превышает границу $C$ (оно «не слишком велико»), то описанный метод успешно справляется с задачей идентификации.

Понятно, что при использовании обсуждаемого метода вероятности ошибок первого и второго рода зависят не только от выбора границы $C$, но и от выбора линейной комбинации $x_{i_{1}} \oplus x_{i_{2}} \oplus \cdots \oplus x_{i_{k}}$. Для минимизации вероятности ошибки первого рода при фиксированном $C$ необходимо выбирать линейную комбинацию (вероятностное линейное соотношение) с наибольшим преобладанием. В настоящей работе предложены алгебраические подходы к поиску вероятностных линейных соотношений на отрезке произвольной двоичной рекурренты.

Пусть $R$ - конечное кольцо с единицей $e, u \in R^{\infty}$ - последовательность над $R, t \in \mathbb{N}, m \in \mathbb{N}_{0}$. Рассмотрим отрезок последовательности $u$ длины $t$ :

$$
u(0), u(1), u(2), \ldots, u(t-1) \text {. }
$$

Набор коэффициентов $\mathbf{a}=\left(a_{0}, a_{1}, \ldots, a_{m-1}, a_{m}\right) \in R^{m+1}$ будем называть вероятностным линейным соотночением (ВЛС) степени $m$ на отрезке (1), если $t>m, a_{m} \neq 0$ и среди значений

$$
a_{0} u(i)+a_{1} u(i+1)+a_{2} u(i+2)+\cdots+a_{m} u(i+m), \quad i=0,1, \ldots, t-m-1,
$$

доля нулей строго больше, чем $|R|^{-1}$. Если сопоставить всякому вектору $\mathbf{a} \in R^{m+1}$ многочлен

$$
f_{\mathbf{a}}(x)=a_{0}+a_{1} x+a_{2} x^{2}+\cdots+a_{m} x^{m} \in R[x],
$$

то определение ВЛС а степени $m$ можно записать так:

$$
a_{m} \neq 0 \quad \text { и } \quad\left|\left\{i \in\{0,1, \ldots, t-m-1\}:\left(f_{\mathbf{a}}(x) \cdot u\right)(i)=0\right\}\right|>\frac{t-m}{|R|} .
$$


В этой ситуации будем говорить, что ВЛС а и многочлен (2) соответствуют друг другу.

Если многочлен $f_{\mathbf{a}}(x)$, соответствующий ВЛС $\mathbf{a}$, является унитарным $\left(a_{m}=e\right)$, то будем называть его вероятностным характеристическим многочленом (ВХМ) отрезка (1) степени $\mathrm{m}$.

Величину

$$
\delta\left(f_{\mathbf{a}}, u, t\right)=\frac{\left|\left\{i \in\{0,1, \ldots, t-m-1\}:\left(f_{\mathbf{a}}(x) \cdot u\right)(i)=0\right\}\right|}{t-m}-\frac{1}{|R|}
$$

назовем преобладанием многочлена $f_{\mathbf{a}}$. Если $\mathbf{a}-$ ВЛС, соответствующее многочлену $f_{\mathbf{a}}(x) \in R[x]$, то величину $\delta\left(f_{\mathbf{a}}, u, t\right)$ будем называть также преобладанием ВЛС а.

В связи с введенными понятиями возникают следующие вопросы:

1. Существуют ли у отрезка (1) ВЛС (ВХМ)? Какова минимальная степень ВЛС на отрезке (1)? Какова минимальная степень ВХМ отрезка (1)?

2. Для данного $m \in \mathbb{N}_{0}$ найти одно или все ВЛС (ВХМ) степени $m$, преобладания которых больше заданного значения $\delta$.

3. Указать алгоритм поиска наилучшего (с точки зрения преобладания $\delta$ ) ВЛС (ВХМ) среди всех ВЛС (ВХМ), степени которых не превосходят $m$.

Нам понадобятся следующие обозначения:

1) $\rho(u, t)$ - глубина сбалансированности отрезка (1) - максимальное такое $\rho \geqslant 0$, что на отрезке (1) появляются все $\rho$-граммы, и все $\rho$-граммы появляются одинаковое число раз,

2) $r(u, t)$ - ранг (линейная сложность) отрезка (1), т. е. минимум длин линейных регистров сдвига над $R$, вырабатывающих отрезок (1) при некотором начальном заполнении,

3) $\mathcal{R}(u, t)$ - минимум степеней всех ВЛС отрезка (1),

4) $r_{p}(u, t)$ - вероятностный ранг отрезка (1) - минимум степеней всех ВXМ отрезка (1),

5) $\bar{r}(u, t)$ - нелинейный ранг (рекурсивная сложность) отрезка (1) - минимум длин регистров сдвига над $R$ (вообще говоря, нелинейных), вырабатывающих отрезок (1) при некотором начальном заполнении. 
Имеет место следующее утверждение.

Утверждение 1. Справедливы неравенства:

a) $\bar{r}(u, t) \leqslant r(u, t)$,

b) $\rho(u, t) \leqslant r_{p}(u, t) \leqslant r(u, t)$,

c) $\mathcal{R}(u, t) \leqslant r_{p}(u, t)$, причем если $R-$ поле, то $\mathcal{R}(u, t)=r_{p}(u, t)$.

Доказательство. Утверждения а) и с) очевидны. Поскольку характеристический многочлен, в частности, является вероятностным характеристическим многочленом, верна верхняя оценка п. b). Докажем нижнюю оценку.

Пусть $\rho=\rho(u, t)$. Покажем, что у отрезка (1) нет ВХМ, степень которых не превосходит $\rho-1$. Предположим противное. Пусть $f(x)-$ ВХМ степени $m \leqslant \rho-1:$

$$
f(x)=a_{0}+a_{1} x+a_{2} x^{2}+\cdots+a_{m-1} x^{m-1}+x^{m} .
$$

На отрезке (1) каждая $(m+1)$-грамма появляется $(t-m) \cdot|R|^{-m-1}$ раз. Количество $(m+1)$-грамм вида

$$
\left(u(i), u(i+1), \ldots, u(i+m-1),-\sum_{j=0}^{m-1} a_{j} u(i+j)\right)
$$

равно, таким образом,

$$
|R|^{m} \cdot(t-m) \cdot \frac{1}{|R|^{m+1}}=(t-m) \cdot \frac{1}{|R|} .
$$

Отсюда

$$
\frac{|\{i \in\{0,1, \ldots, t-m-1\}:(f(x) \cdot u)(i)=0\}|}{t-m}-\frac{1}{|R|}=0,
$$

что противоречит определению ВХМ $f$.

В данной работе для случая $R=\mathbb{Z}_{2}$ предложен ряд алгоритмов построения ВЛС (BХМ) и вычислены их трудоемкости. Перечислим полученные результаты. 
1. Пусть $m-$ нелинейный ранг (рекурсивная сложность) отрезка (1). В разделе 2 предложен алгоритм вычисления всех ВХМ отрезка (1), степень которых строго меньше $m$. Трудоемкость алгоритма равна $O\left(m 2^{m}\right)$; основной вклад в трудоемкость дает этап, связанный с быстрым преобразованием Фурье (теорема 7).

2. В разделе 3 исследуется вычислительная сложность построения весовой функции Хемминга $W_{\mathcal{K}}(x, y)$ линейного рекурсивного кода $\mathcal{K}=$ $L_{R}^{\overline{0, n-1}}(F)$, где

$$
\operatorname{deg} F=m<n \leqslant T(F)
$$

(см., например, [8]). Показано (теорема 11), что функция $W_{\mathcal{K}}(x, y)$ может быть вычислена с трудоемкостью $m 2^{m}(1+o(1))$ операций в поле $\mathbb{Q}$.

3. В разделе 4 предложен полиномиальный относительно $t$ алгоритм построения одного ВХМ отрезка (1), в основу которого положен метод ортогонализации Грама-Шмидта [2].

4. Пусть минимальный многочлен $F(x)$ последовательности $u$ реверсивен и сепарабелен, $S=G F\left(2^{m}\right)$ - минимальное поле разложения $F(x)$,

$$
\operatorname{deg} F(x)=\mu, \quad \tau=2^{m}-1 .
$$

В разделе 5 предложен такой алгоритм построения ЛРП $v \in R^{\infty}$, что rang $v \leqslant m$ и

$$
\mathbb{P}\{u(i)=v(i)\}>\frac{1}{2}
$$

при случайном и равновероятном выборе $i \in\{0,1, \ldots, \tau-1\}$. Трудоемкость предложенного алгоритма есть $O\left(m 2^{m}\right)$ (теорема 17). Его модификация в ряде случаев позволяет строить один ВХМ отрезка (1) длины $t=\tau+\mu-1$, преобладание которого не менее

$$
\frac{2^{m / 2}-1}{2^{m+1}-2}
$$

(следствие 18). Трудоемкость этой модификации есть $O\left(\mu^{3}+m 2^{m}\right)$.

В следующем разделе обсуждается возможность использования быстрого преобразования Фурье для построения ВХМ. 


\section{2. Алгоритм, использующий преобразование Фурье}

Пусть $R=\mathbb{Z}_{2}, u$ - линейная рекуррентная последовательность, $\bar{r}(u, t)$ - нелинейный ранг отрезка (1), $t>m \geqslant \bar{r}(u, t)$. В этом разделе обсуждается простейший алгоритм поиска всех ВХМ отрезка (1), степень которых не превосходит $m$. Алгоритм представляет собой обычную процедуру маркировки зацепленных $(m+1)$-грамм с последующей обработкой полученных результатов методом быстрого преобразования Фурье.

Опишем алгоритм. Сначала построим по отрезку (1) совокупность $(m+1)$ грамм

$$
(u(i), u(i+1), \ldots, u(i+m-1), u(i+m)), \quad i=0,1, \ldots, t-m-1 .
$$

Отрезку (1) и значению $m$ сопоставим булеву функцию

$$
\chi_{u, t, m}: R^{m+1} \rightarrow R,
$$

определенную условием: для вектора $\left(a_{0}, a_{1}, \ldots, a_{m}\right) \in R^{m+1}$ равенство

$$
\chi_{u, t, m}\left(a_{0}, a_{1}, \ldots, a_{m}\right)=0
$$

выполняется тогда и только тогда, когда вектор $\left(a_{0}, a_{1}, \ldots, a_{m}\right)$ принадлежит семейству (3).

Пусть $\Lambda(u)$ и $T(u)$ - соответственно длина подхода и период последовательности $u$ (см., например, [2]).

Лемма 2. Если $t=\Lambda(u)+T(u)+m$, то на отрезке $(1)$ все $(m+1)$-граммы попарно различны, а семейство (3) совпадает с множеством всех $(m+1)$ грамм последовательности и.

Доказательство. Поскольку $m \geqslant \bar{r}(u, t)$, на отрезке $u(0,1, \ldots, t-2)$ все $m$-граммы попарно различны. Следовательно, на отрезке (1) имеется ровно одна пара повторяющихся $m$-грамм:

$$
u(\lambda, \lambda+1, \ldots, \lambda+m-1)=u(\lambda+\tau, \lambda+\tau+1, \ldots, \lambda+\tau+m-1),
$$

где $\lambda=\Lambda(u), \quad \tau=T(u)$. Поэтому если на отрезке (1) есть повторяющиеся $(m+1)$-граммы, то

$$
u(\lambda-1, \lambda, \ldots, \lambda+m-1)=u(\lambda+\tau-1, \lambda+\tau, \ldots, \lambda+\tau+m-1),
$$

и, в частности, $u(\lambda-1)=u(\lambda+\tau-1)$, что противоречит определению длины подхода. 
Докажем вторую часть утверждения. От противного: пусть $(m+1)$-грамма

$$
u(i, i+1, \ldots, i+m)
$$

не принадлежит множеству (3). Тогда $i \geqslant \lambda+\tau$ и $(m+1)$-грамма (4) лежит на цикле последовательности $u$. Однако все $(m+1)$-граммы, лежащие на цикле последовательности $u$, принадлежат множеству (3). Противоречие.

Будем обозначать знаком $\|\cdot\|$ вес булевой функции. Имеет место следующее утверждение.

Утверждение 3. Если $t \leqslant \Lambda(u)+T(u)+m$, то функции $\chi_{u, t, m}$ однозначно определяет отрезок (1). Если $t \geqslant \Lambda(u)+T(u)+m$, то функциия $\chi_{u, t, m}$ однозначно определяет последовательность $u$.

Доказательство. 1. Пусть $t \leqslant \Lambda(u)+T(u)+m$. По лемме 2 в семействе (3) все $(m+1)$-граммы попарно различны. Поэтому $\left\|\chi_{u, t, m}\right\|=2^{m+1}-t+m$, и, поскольку арность функции $\chi_{u, t, m}$ равна $m+1$, функция $\chi_{u, t, m}$ однозначно определяет длину $t$ отрезка (1).

Если $t \leqslant \Lambda(u)+T(u)+m$, то все $m$-граммы вида

$$
(u(i), u(i+1), \ldots, u(i+m-1)), \quad i=0,1, \ldots, t-m,
$$

попарно различны. Заметим, что в множестве $(m+1)$-грамм (3) существует единственная $(m+1)$-грамма $\mathbf{a}=\left(a_{0}, a_{1}, \ldots, a_{m}\right)$ со свойством: ни для какого $\alpha \in R(m+1)$-грамма $\left(\alpha, a_{0}, a_{1}, \ldots, a_{m-1}\right)$ не принадлежит множеству (3) (о такой $(m+1)$-грамме а будем говорить, что она не достраивается слева). Действительно, в качестве а можно взять $\mathbf{u}=(u(0), u(1), \ldots, u(m)):$ если $(\alpha, u(0), u(1), \ldots, u(m-1))$ принадлежит множеству (3), то в семействе (5) по меньшей мере дважды встречается $m$-грамма $(u(0), u(1), \ldots, u(m-1))$, что невозможно. Если же, наряду с $\mathbf{u}$, в множестве (3) есть еще одна $(m+1)$ грамма a, которая не достраивается слева, то

$$
\mathbf{a}=(u(i), u(i+1), \ldots, u(i+m)), \quad i \geqslant 1 .
$$

Тогда $(m+1)$-грамма $\left(u(i-1), a_{0}, a_{1}, \ldots, a_{m-1}\right)$ принадлежит множеству (3), т. е. а достраивается слева. Противоречие.

Далее, заметим, что если $(m+1)$-грамма а принадлежит множеству (3) и отлична от $(u(t-m-1), u(t-m), \ldots, u(t-1))$, то существует единственное значение $\varphi(\mathbf{a}) \in R$, при котором вектор $\left(a_{1}, a_{2}, \ldots, a_{m}, \varphi(\mathbf{a})\right)$ принадлежит множеству (3) (будем говорить, что а достраивается справа значением $\varphi(\mathbf{a}))$. 
Действительно, всякая $(m+1)$-грамма

$$
(u(i), u(i+1), \ldots, u(i+m)), \quad i<t-m-1,
$$

достраивается справа значением $u(i+m+1)$. Если же обе $(m+1)$-граммы

$$
\left(a_{1}, a_{2}, \ldots, a_{m}, 0\right) \quad \text { и } \quad\left(a_{1}, a_{2}, \ldots, a_{m}, 1\right)
$$

принадлежат множеству (3), то $m$-грамма $\left(a_{1}, a_{2}, \ldots, a_{m}\right)$ по меньшей мере дважды встречается в семействе (5). Противоречие.

Для всех точек $\mathbf{a} \in R^{m+1}$, не принадлежащих множеству (3), а также для $\mathbf{a}=(u(t-m-1), u(t-m), \ldots, u(t-1))$ положим $\varphi(\mathbf{a})$ равным произвольному значению из множества $R$. Таким образом, построена $(m+1)$-местная булева функция $\varphi$. Получаем следующий алгоритм построения отрезка (1) на основе функции $\chi_{u, t, m}$.

- Найти по функции $\chi_{u, t, m}$ значение $t$.

- Найти в множестве (3) ту единственную $(m+1)$-грамму а, которая не достраивается слева.

- Используя а в качестве начального заполнения регистра сдвига с функцией обратной связи $\varphi$, выработать с помощью этого регистра отрезок длины $t$. Он совпадет с отрезком (1).

2. Пусть $t \geqslant \Lambda(u)+T(u)+m, t_{0}=\Lambda(u)+T(u)+m$. Из леммы 2 следует, что $\chi_{u, t, m}=\chi_{u, t_{0}, m}$, поэтому достаточно доказать утверждение лишь для $t=t_{0}$. Согласно п. 1 по функции $\chi_{u, t_{0}, m}$ однозначно восстанавливается отрезок $\left(u(0), u(1), \ldots, u\left(t_{0}-1\right)\right)$. Зная $m \geqslant \bar{r}(u, t)$ и первые $\Lambda(u)+T(u)+m$ знаков рекурренты $u$, нетрудно восстановить всю рекурренту $u$.

В связи с утверждением 3 всюду далее будем считать, что

$$
t \leqslant \Lambda(u)+T(u)+m .
$$

Это ограничение оправданно, поскольку вся последовательность $u$ на практике «почти никогда» не бывает известна.

Если $\mathbf{a}=\left(a_{0}, a_{1}, \ldots, a_{m}\right), \mathbf{x}=\left(x_{1}, x_{2}, \ldots, x_{m+1}\right)$, то линейную булеву функцию

$$
a_{0} x_{1} \oplus a_{1} x_{2} \oplus \cdots \oplus a_{m} x_{m+1}
$$

коротко обозначим через $(\mathbf{a}, \mathbf{x})$. Будем искать статистические аналоги характеристической функции $\chi_{u, t, m}(\mathbf{x})$ в классе линейных функций $(\mathbf{a}, \mathbf{x})$, $\mathbf{a} \in R^{m+1}$. 
Назовем преобладанием линейного статистического аналога $(\mathbf{a}, \mathbf{x})$ величину $\delta_{\mathbf{a}}(u, t)$, определенную равенством

$$
2^{m+1} \delta_{\mathbf{a}}(u, t)=2^{m}-\left\|(\mathbf{a}, \mathbf{x}) \oplus \chi_{u, t, m}(\mathbf{x})\right\| .
$$

Пусть $\theta-$ нулевая вектор-строка длины $m+1$. Связь преобладаний $\delta_{\mathbf{a}}(u, t)$ и $\delta\left(f_{\mathbf{a}}, u, t\right)$ устанавливает следующее утверждение.

Утверждение 4. Если выполнено условие (6) $u \mathbf{a} \neq \theta$, то

$$
\delta\left(f_{\mathbf{a}}, u, t\right)=\frac{2^{m}}{t-m} \cdot \delta_{\mathbf{a}}(u, t) .
$$

Доказательство. Пусть $V_{0}$ есть совокупность (3), а $V_{1}=R^{m+1} \backslash V_{0}$. Так как $\mathbf{a} \neq \theta$, то функция $(\mathbf{a}, \mathbf{x})$ равновероятна на множестве $R^{m+1}$. Если в множестве $V_{0}$ имеется $k \geqslant 0$ векторов, на которых функция $(\mathbf{a}, \mathbf{x})$ принимает значение 0 , то количество векторов $\mathbf{x} \in V_{1}$, на которых $(\mathbf{a}, \mathbf{x})=1$, равно

$$
\left|V_{1}\right|-\left(2^{m}-k\right)=2^{m+1}-\left|V_{0}\right|-2^{m}+k=2^{m}-t+m+k .
$$

Ввиду (7) имеем:

$$
\begin{aligned}
\delta_{\mathbf{a}}(u, t) & =\frac{1}{2}-\frac{1}{2^{m+1}} \cdot\left\|(\mathbf{a}, \mathbf{x}) \oplus \chi_{u, t}(\mathbf{x})\right\|= \\
& =\frac{1}{2}-\frac{1}{2^{m+1}} \cdot\left((t-m-k)+\left(2^{m}-k\right)\right)=\frac{1}{2^{m+1}}(2 k-t+m) .
\end{aligned}
$$

Так как

получим:

$$
\delta\left(f_{\mathbf{a}}, u, t\right)=\frac{k}{t-m}-\frac{1}{2} \quad \text { и } \quad t>m
$$

$$
\begin{aligned}
\delta_{\mathbf{a}}(u, t) & =\frac{1}{2^{m+1}}(2 k-t+m)=\frac{t-m}{2^{m+1}} \cdot \frac{1}{t-m}(2 k-t+m)= \\
& =\frac{t-m}{2^{m+1}} \cdot 2\left(\frac{k}{t-m}-\frac{1}{2}\right)=\frac{t-m}{2^{m}} \cdot \delta\left(f_{\mathbf{a}}, u, t\right) .
\end{aligned}
$$

Равенство (8) доказано.

Обозначим через $\Delta_{\mathbf{a}}\left(\chi_{u, t, m}\right)$ коэффициент статистической структуры функции $\chi_{u, t, m}$, соответствующий вектору $\mathbf{a}$, т. е. значение

$$
\Delta_{\mathbf{a}}\left(\chi_{u, t, m}\right)=2^{m}-\left\|(\mathbf{a}, \mathbf{x}) \oplus \chi_{u, t, m}(\mathbf{x})\right\|
$$

(см., например, [4]). Из равенства (7) вытекает, что $\Delta_{\mathbf{a}}\left(\chi_{u, t, m}\right)=2^{m+1} \delta_{\mathbf{a}}(u, t)$. Во введенных обозначениях утверждение 4 можно переформулировать так. 
Следствие 5. Если выполнено условие (6) $u$ a $\neq \theta$, то

$$
\delta\left(f_{\mathbf{a}}, u, t\right)=\frac{\Delta_{\mathbf{a}}\left(\chi_{u, t, m}\right)}{2(t-m)} .
$$

Очевидно, $\delta\left(f_{\theta}, u, t\right)=1 / 2$.

Установим некоторые свойства чисел (9).

Утверждение 6. Пусть выполнено неравенство (6). Тогда:

1) верно равенство

$$
\sum_{\mathbf{a} \in R^{m+1}} \delta\left(f_{\mathbf{a}}, u, t\right)^{2}=\frac{2^{m-1}}{t-m}
$$

2) если $(u(t-m-1), u(t-m), \ldots, u(t-1)) \neq \theta$, то

$$
\sum_{\mathbf{a} \in R^{m+1}} \delta\left(f_{\mathbf{a}}, u, t\right)=0
$$

3) если $(u(t-m-1), u(t-m), \ldots, u(t-1))=\theta$, то

$$
\sum_{\mathbf{a} \in R^{m+1}} \delta\left(f_{\mathbf{a}}, u, t\right)=\frac{2^{m}}{t-m}
$$

4) для всякого $\mathbf{a} \in R^{m+1}$ верно равенство

$$
\sum_{\mathbf{b} \in R^{m+1}} \delta\left(f_{\mathbf{b}}, u, t\right) \cdot \delta\left(f_{\mathbf{a} \oplus \mathbf{b}}, u, t\right)=\delta\left(f_{\mathbf{a}}, u, t\right) \cdot \frac{2^{m}}{t-m} .
$$

Доказательство. Ввиду (9), цепочки соотношений

$$
\Delta_{\theta}\left(\chi_{u, t, m}\right)=2^{m}-\left\|\chi_{u, t, m}\right\|=2^{m}-\left(2^{m+1}-t+m\right)=t-m-2^{m}
$$

и равенства Парсеваля для булевой функции $\chi_{u, t, m}$ имеем:

$$
\begin{aligned}
2^{2 m} & =\left(t-m-2^{m}\right)^{2}+\sum_{\mathbf{a} \neq \theta}\left(2(t-m) \delta\left(f_{\mathbf{a}}, u, t\right)\right)^{2}= \\
& =\left(t-m-2^{m}\right)^{2}+4(t-m)^{2} \sum_{\mathbf{a} \neq \theta} \delta\left(f_{\mathbf{a}}, u, t\right)^{2}
\end{aligned}
$$


Учитывая, что $\delta\left(f_{\theta}, u, t\right)=1 / 2$, получаем:

$$
\sum_{\mathbf{a} \in R^{m+1}} \delta\left(f_{\mathbf{a}}, u, t\right)^{2}=\frac{2^{2 m}-\left(2^{m}-(t-m)\right)^{2}}{4(t-m)^{2}}+\frac{1}{4}=\frac{2^{m-1}}{t-m} .
$$

Равенство (10) доказано.

Докажем равенства (11) и (12). Ввиду известного равенства для суммы коэффициентов статистической структуры (см., например, [4]), а также соотношений (9) и (14) имеем:

$$
2^{m}(-1)^{\chi u, t, m(\theta)}=t-m-2^{m}+2(t-m) \sum_{\mathbf{a} \neq \theta} \delta\left(f_{\mathbf{a}}, u, t\right) .
$$

Тогда, поскольку $\delta\left(f_{\theta}, u, t\right)=1 / 2$,

$$
\sum_{\mathbf{a} \in R^{m+1}} \delta\left(f_{\mathbf{a}}, u, t\right)=\frac{2^{m}(-1)^{\chi u, t, m}(\theta)-t+m+2^{m}}{2(t-m)}+\frac{1}{2}=\frac{2^{m-1}}{t-m}\left(1+(-1)^{\chi_{u, t, m}(\theta)}\right) .
$$

Осталось показать, что равенство $\chi_{u, t, m}(\theta)=0$ имеет место тогда и только тогда, когда

$$
(u(t-m-1), u(t-m), \ldots, u(t-1))=\theta .
$$

Достаточность очевидна: из равенства (15) следует, что $\chi_{u, t, m}(\theta)=0$. Докажем необходимость. Поскольку $m \geqslant \bar{r}(u, t)$, из того, что (15) не выполняется, следует, что в совокупности (3) нет ни одной нулевой $(m+1)$-граммы, т. е. $\chi_{u, t, m}(\theta)=1$.

Докажем формулу (13). При $\mathbf{a}=\theta$ формула (13) превращается в формулу (10). Пусть $\mathbf{a} \neq 0$. Ввиду равенств $\delta\left(f_{\theta}, u, t\right)=1 / 2$, (9) и (11) имеем:

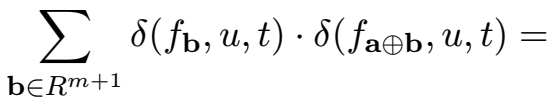

$$
\begin{aligned}
& =\delta\left(f_{\theta}, u, t\right) \delta\left(f_{\mathbf{a}}, u, t\right)+\sum_{\mathbf{b} \neq \theta} \delta\left(f_{\mathbf{b}}, u, t\right) \cdot \delta\left(f_{\mathbf{a} \oplus \mathbf{b}}, u, t\right)= \\
& =\frac{\Delta_{\mathbf{a}}\left(\chi_{u, t, m}\right)}{4(t-m)}+\sum_{\mathbf{b} \neq \theta, \mathbf{b} \neq \mathbf{a}} \delta\left(f_{\mathbf{b}}, u, t\right) \cdot \delta\left(f_{\mathbf{a} \oplus \mathbf{b}}, u, t\right)+\delta\left(f_{\theta}, u, t\right) \delta\left(f_{\mathbf{a}}, u, t\right)=
\end{aligned}
$$

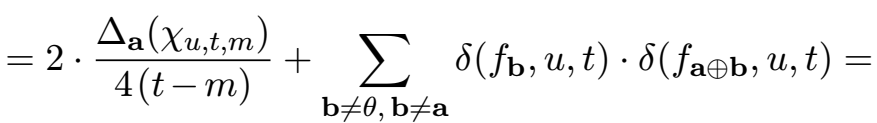

$$
\begin{aligned}
& =\frac{\Delta_{\mathbf{a}}\left(\chi_{u, t, m}\right)}{2(t-m)}+\frac{1}{4(t-m)^{2}} \sum_{\mathbf{b} \neq \theta, \mathbf{b} \neq \mathbf{a}} \Delta_{\mathbf{b}}\left(\chi_{u, t, m}\right) \Delta_{\mathbf{a} \oplus \mathbf{b}}\left(\chi_{u, t, m}\right) .
\end{aligned}
$$


По свойству коэффициентов статистической структуры (см., например, [4]) при $\mathbf{a} \neq \theta$ верно равенство

$$
\sum_{\mathbf{b} \neq \theta, \mathbf{b} \neq \mathbf{a}} \Delta_{\mathbf{b}}\left(\chi_{u, t, m}\right) \Delta_{\mathbf{a} \oplus \mathbf{b}}\left(\chi_{u, t, m}\right)=-2 \Delta_{\mathbf{a}}\left(\chi_{u, t, m}\right) \Delta_{\theta}\left(\chi_{u, t, m}\right) .
$$

Отсюда с учетом (14) получаем:

$$
\begin{aligned}
& \sum_{\mathbf{b} \in R^{m+1}} \delta\left(f_{\mathbf{b}}, u, t\right) \cdot \delta\left(f_{\mathbf{a} \oplus \mathbf{b}}, u, t\right)= \\
& =\frac{\Delta_{\mathbf{a}}\left(\chi_{u, t, m}\right)}{2(t-m)}\left(1-\frac{\Delta_{\theta}\left(\chi_{u, t, m}\right)}{t-m}\right)=\delta\left(f_{\mathbf{a}}, u, t\right) \cdot \frac{2^{m}}{t-m} .
\end{aligned}
$$

Формула (10) является аналогом равенства Парсеваля. Теперь докажем центральный результат этого раздела.

Теорема 7. Пусть $m \geqslant \bar{r}(u, t)$. При условии (6) набор чисел

$$
\delta\left(f_{\mathbf{a}}, u, t\right), \quad \mathbf{a} \in R^{m+1},
$$

однозначно определяет отрезок (1). По отрезку (1) и значению $m$ набор чисел (16) вычисляется за $O\left(m 2^{m}\right)$ операчий в поле $\mathbb{Q}$.

Доказательство. Количество чисел в наборе (16) равно $2^{m+1}$, поэтому по набору (16) однозначно восстанавливается значение $m$. Зная $m$ и набор (16), можно, исходя из равенства (10), найти длину $t$ отрезка (1). Тогда, пользуясь равенствами (9) и (14), по набору (16) можно вычислить набор коэффициентов статистической структуры функции $\chi_{u, t, m}$, а значит, и саму функцию $\chi_{u, t, m}$. Согласно утверждению 3 при условии (6) по функции $\chi_{u, t, m}$ однозначно восстанавливается отрезок (1).

Пусть теперь известны отрезок (1) и значение $m \geqslant \bar{r}(u, t)$. За $t-m \leqslant 2^{m}$ операций обращения в память по адресу можно построить по отрезку (1) и значению $m$ таблицу истинности функции $\chi_{u, t, m}$. Методом быстрого преобразования Фурье за $O\left(m 2^{m}\right)$ операций в кольце $\mathbb{Z}$ вычисляется набор коэффициентов статистической структуры функции $\chi_{u, t, m}$. Зная этот набор, $m$ и $t$, можно с помощью равенства (9) вычислить набор чисел (16) за $O\left(2^{m}\right)$ операций в поле $\mathbb{Q}$. 
Утверждение 8. При условии $t<2^{m}+m$ отрезок (1) имеет BXM, степень которого не превосходит $\bar{r}(u, t)$. В частности, $r_{p}(u, t) \leqslant \bar{r}(u, t)$.

Доказательство. Достаточно доказать существование такого вектора $\mathbf{a} \neq \theta$, что $\delta\left(f_{\mathbf{a}}, u, t\right)>0$ или, что эквивалентно, $\Delta_{\mathbf{a}}\left(\chi_{u, t, m}\right)>0$.

Заметим, что

$$
\Delta_{\theta}\left(\chi_{u, t, m}\right)=t-m-2^{m}<0
$$

ввиду (14). Если все значения $-\Delta_{\mathbf{a}}\left(\chi_{u, t, m}\right), \mathbf{a} \in R^{m+1}$, неотрицательны, то

$$
\left(\sum_{\mathbf{a} \in R^{m+1}} \Delta_{\mathbf{a}}\left(\chi_{u, t, m}\right)\right)^{2} \geqslant \sum_{\mathbf{a} \in R^{m+1}} \Delta_{\mathbf{a}}\left(\chi_{u, t, m}\right)^{2},
$$

причем равенство возможно тогда и только тогда, когда ровно один коэффициент статистической структуры равен нулю. Левая и правая части неравенства (17) равны $2^{2 m}$. Поскольку $\Delta_{\theta}\left(\chi_{u, t, m}\right)<0$, коэффициент $\Delta_{\theta}\left(\chi_{u, t, m}\right)$ является единственным ненулевым коэффициентом статистической структуры. Но тогда, поскольку

$$
\Delta_{\theta}\left(\chi_{u, t, m}\right)^{2}<2^{2 m}
$$

имеем:

$$
\sum_{\mathbf{a} \in R^{m+1}} \Delta_{\mathbf{a}}\left(\chi_{u, t, m}\right)^{2}<2^{2 m}
$$

что противоречит равенству Парсеваля. Итак, существует вектор $\mathbf{a} \in R^{m+1}$, для которого $\Delta_{\mathbf{a}}\left(\chi_{u, t, m}\right)>0$. Поскольку $\Delta_{\theta}\left(\chi_{u, t, m}\right)<0$, этот вектор $\mathbf{a}-$ ненулевой.

Отметим, что для идентификации автономных двоичных регистров сдвига по выходу представляют интерес не только векторы $\mathbf{a} \in R^{m+1}$, для которых $\delta\left(f_{\mathbf{a}}, u, t\right)>0$, но и векторы $\mathbf{a} \in R^{m+1}$, для которых $\delta\left(f_{\mathbf{a}}, u, t\right)<0$. Вектор $\mathbf{a}$, для которого $\delta\left(f_{\mathbf{a}}, u, t\right)<0$, не является ВЛС в смысле нашего определения (см. раздел 1), поэтому требуется другое, более широкое понятие.

Пусть $m=\bar{r}(u, t)$. Вектор $\mathbf{a} \in R^{m+1}$ назовем неравновероятной линейной комбинацией (НЛК), если $\delta\left(f_{\mathbf{a}}, u, t\right) \neq 0$. Вес $\|\mathbf{a}\|$ НЛК а назовем длиной НЛК а. Пусть

$$
w_{p}(u, t)=\min \left\{\|\mathbf{a}\|: \delta\left(f_{\mathbf{a}}, u, t\right) \neq 0 \text { и } \mathbf{a} \neq \theta\right\} .
$$

Справедлива следующая теорема, которая может применяться в переборных алгоритмах поиска НЛК. 
Теорема 9. Пусть $m=\bar{r}(u, t), t=m+n$ и выполняется неравенство (6). Тогда

$$
w_{p}(u, t) \leqslant l_{2}(n),
$$

где $l_{2}(n)$ - число разрядов в двоичной записи числа $n$.

Доказательство. Пусть $\operatorname{cor}\left(\chi_{u, t, m}\right)$ равно такому максимальному $k \geqslant 1$, что функция $\chi_{u, t, m}$ является корреляционно-имунной порядка $k$, если такое $k$ существует, и равно нулю в противном случае. Согласно неравенству Зигенталера [7] имеем:

$$
\operatorname{cor}\left(\chi_{u, t, m}\right)+\operatorname{deg}\left(\chi_{u, t, m}\right) \leqslant m+1 .
$$

Ввиду [4, теорема 5.3] значение $\operatorname{cor}\left(\chi_{u, t, m}\right)$ равно максимальному $k \geqslant 1$ со свойством

$$
\forall \mathbf{a} \in R^{m+1}:(1 \leqslant\|\mathbf{a}\| \leqslant k) \Rightarrow\left(\Delta_{\mathbf{a}}\left(\chi_{u, t, m}\right)=0\right),
$$

если такое $k$ существует, и равно нулю в противном случае. Ввиду равенства (9) имеем:

$$
w_{p}(u, t)=\operatorname{cor}\left(\chi_{u, t, m}\right)+1 .
$$

Из (19) и (20) получаем:

$$
w_{p}(u, t) \leqslant m+2-\operatorname{deg}\left(\chi_{u, t, m}\right) .
$$

Пусть $d=\operatorname{deg}\left(\chi_{u, t, m}\right)$. Используя известные соотношения между весом и степенью нелинейности булевой функции (см., например, [3, задача 8.33]), имеем:

$$
2^{m+1-d} \leqslant\left\|\chi_{u, t, m}\right\| \leqslant 2^{m+1}-2^{m+1-d} .
$$

Поскольку $\left\|\chi_{u, t, m}\right\|=2^{m+1}-n$, верно неравенство

$$
2^{m+1-d} \leqslant \min \left\{n, 2^{m+1}-n\right\},
$$

откуда

$$
m+1-d \leqslant \log _{2} \min \left\{n, 2^{m+1}-n\right\} .
$$

Поэтому ввиду (21) справедливо неравенство

$$
w_{p}(u, t) \leqslant 1+\log _{2} \min \left\{n, 2^{m+1}-n\right\} .
$$

Из неравенства (6) следует, что $n \leqslant T(u) \leqslant 2^{m}$, поэтому

$$
1+\log _{2} \min \left\{n, 2^{m+1}-n\right\}=1+\log _{2} n=l_{2}(n) .
$$

Неравенство (18) доказано. 
Следствие 10. В условиях теоремь 9 существует алгоритм поиска кратчайшей неравновероятной линейной комбинации $\mathbf{a} \in R^{m+1} \backslash\{\theta\}$, имеющий трудоемкость

$$
O\left(n \cdot \sum_{k=1}^{l_{2}(n)} k C_{m+1}^{k}\right)
$$

операчий в $R$, где $l_{2}(n)$ - число разрядов в двоичной записи числа $n$.

Доказательство. Будем перебирать все векторы $\mathbf{a} \in R^{m+1}$ в порядке возрастания их веса: сначала перебираем векторы веса 1, затем векторы веса 2 и т. д. В конце перебираем все векторы веса $l_{2}(n)$. Согласно теореме 9 среди перебираемых векторов обязательно найдется кратчайшая НЛК. Для каждого из перебираемых векторов а вычисляем значение $\delta\left(f_{\mathbf{a}}, u, t\right)$. Первый вектор $\mathbf{a}$, для которого $\delta\left(f_{\mathbf{a}}, u, t\right) \neq 0$, будет являться кратчайшей НЛК. Если $\|\mathbf{a}\|=k$, то величина $\delta\left(f_{\mathbf{a}}, u, t\right)$ может быть вычислена за $O(n k)$ операций в $R$. Поскольку число векторов $\mathbf{a} \in R^{m+1}$ веса $k$ равно $C_{m+1}^{k}$, трудоемкость предложенного алгоритма выражается формулой (22).

В этом разделе описан метод, позволяющий строить по отрезку (1) все $\mathrm{BXM}$, степень которых не превосходит значения $m \geqslant \bar{r}(u, t)$, и вычислять соответствующие преобладания. В следующем разделе этот метод будет использован для вычисления весовой функции так называемого линейного рекурсивного кода (ЛРК).

\section{3. О вычислении весовой функции ЛРК}

Пусть $F(x) \in R[x]$ - реверсивный многочлен степени $m \geqslant 1, T=T(F)$ - его период, $m<n \leqslant T$, и код $\mathcal{K}$ определен равенством

$$
\mathcal{K}=L_{R}^{\overline{0, n-1}}(F)=\left\{(u(0), u(1), \ldots, u(n-1)) \mid u \in L_{R}(F)\right\} .
$$

Очевидно, что $\mathcal{K}-$ линейный код. Его называют линейным рекурсивным кодом (ЛРК). Согласно [8] весовой функиией Хемминга $W_{\mathcal{K}}(x, y)$ называется многочлен над $\mathbb{Z}$ вида

$$
W_{\mathcal{K}}(x, y)=\sum_{\mathbf{a} \in \mathcal{K}} x^{n-\|\mathbf{a}\|} y^{\|\mathbf{a}\|}
$$

где $\|\mathbf{a}\|$ есть вес вектора $\mathbf{a}$, т. е. количество единиц в этом векторе.

Линейный рекурсивный код допускает эффективную аппаратную реализацию и поэтому является весьма привлекательным с точки зрения его использования в целях помехоустойчивого кодирования. Корректирующие способности ЛРК определяются его весовой функцией Хемминга. 
Вычисление весовой функции ЛРК (и даже вычисление расстояния ЛРК) - сложная комбинаторная задача, весьма далекая от решения. На данный момент известны лишь отдельные классы ЛРК, для которых получены соответствующие результаты. Так, например, в работе [5] полностью описаны линейные рекурсивные МДР-коды, т. е. линейные рекурсивные коды с расстоянием $n-m+1$. В ряде случаев методом тригонометрических сумм удается исследовать свойства весовой функции линейного рекурсивного ииклического кода с неприводимым многочленом $F(x)$ (см., например, [9]). В данном разделе будет предложен субэкспоненциальный относительно $m$ алгоритм вычисления весовой функции ЛРК, трудоемкость которого существенно ниже трудоемкости тотального перебора.

«Наивный» способ вычисления весовой функции $W_{\mathcal{K}}(x, y)$ требует $n \cdot 2^{m}$ операций в поле $\mathbb{Q}$ (количество слов в коде $\mathcal{K}$ равно $2^{m}$; вес каждого из них вычисляется за $n$ операций в поле $\mathbb{Q}$ ). Следующая теорема позволяет вычислять весовую функцию с трудоемкостью быстрого преобразования Фурье (БПФ). Этот способ асимптотически (при $m \rightarrow \infty$ ) быстрее «наивного» способа.

Теорема 11. Пусть $F(x) \in R[x]-$ реверсивный многочлен степени $m u$ периода T. Если $m<n \leqslant T$, то вычисление весовой функции Хемминга линейного рекурсивного кода $L_{R}^{\overline{0, n-1}}(F)$ может быть осуществлено за

$$
m 2^{m}(1+o(1)), \quad m \rightarrow \infty,
$$

операчий в поле $\mathbb{Q}$.

Доказательство. Рассмотрим отрезок импульсной последовательности $u=e^{F}$, состоящий из ее первых $t=n+m$ знаков (см., например, [2]):

$$
\left(e^{F}(0), e^{F}(1), \ldots, e^{F}(t-1)\right) .
$$

Очевидно, $m \geqslant r(u, t) \geqslant \bar{r}(u, t)$, и выполнено условие (6):

$$
t=n+m \leqslant T+m=\Lambda(u)+T(u)+m .
$$

Из доказательства теоремы 7 следует, что по отрезку (24) можно найти набор коэффициентов (16), осуществив при этом

$$
\left(2^{m+1}+n\right)+(m+1) 2^{m+1}+2^{m+1}=2 m 2^{m}(1+o(1))
$$

операций в поле $\mathbb{Q}$. Здесь первое слагаемое соответствует формированию таблицы функции $\chi_{u, t, m}$ (сначала заполняем всю таблицу единицами, а затем заменяем $n$ единиц нулями), второе слагаемое - БПФ, третье слагаемое получению коэффициентов (16) по формуле (9). 
Рассмотрим набор коэффициентов

$$
\Delta=\left\{\delta\left(f_{\mathbf{a}}, u, t\right) \mid a_{m}=0\right\} .
$$

Если векторы $\mathbf{a} \in R^{m+1}$ упорядочены в лексикографическом порядке, то $\Delta$ есть «первая половина» вектора коэффициентов (16). Исходя из табличного способа оформления алгоритма БПФ (см., например, [1]) легко понять, что набор $\Delta$ можно найти, осуществив

$$
\left(2^{m+1}+n\right)+2^{m}+m 2^{m}+2^{m}=m 2^{m}(1+o(1))
$$

операций в поле $\mathbb{Q}$. Здесь первое слагаемое соответствует формированию таблицы функции $\chi_{u, t, m}$, второе слагаемое - сложению двух половин полученной таблицы, третье слагаемое - БПФ, примененному к верхней половине таблицы, четвертое слагаемое - получению набора коэффициентов $\Delta$ по формуле (9).

Известно (см., например, [2]), что для всякой последовательности $v \in L_{R}(F)$ существует такой вектор $\mathbf{a} \in R^{m+1}$, что $a_{m}=0$ и верно равенство

$$
v=f_{\mathbf{a}}(x) \cdot e^{F}
$$

(здесь многочлен $f_{\mathbf{a}}(x)$ есть генератор последовательности $v$ ). Зная начальный отрезок (24) и соответствующий многочлен $f_{\mathbf{a}}(x)$, можно найти отрезок

$$
(v(0), v(1), \ldots, v(n-1))
$$

последовательности $v$. При этом количество нулей на отрезке (25) вычисляется по формуле

$$
\left(\frac{1}{2}+\delta\left(f_{\mathbf{a}}, u, t\right)\right) \cdot n
$$

Таким образом, за $2 \cdot 2^{m}=o\left(m 2^{m}\right)$ операций в поле $\mathbb{Q}$ по набору $\Delta$ вычисляется совокупность весов всех кодовых слов из кода $\mathcal{K}=L_{R}^{\overline{0, n-1}}(F)$. Располагая этой совокупностью, можно за $2^{m}=o\left(m 2^{m}\right)$ операций найти весовую функцию Хемминга $W_{\mathcal{K}}(x, y)$.

Метод, описанный в доказательстве теоремы 11, особенно эффективен для вычисления весовой функции линейного рекурсивного изикического кода: «наивный» способ требует $O\left(2^{2 m}\right)$ операций, в то время как применение БПФ понижает трудоемкость до $O\left(m 2^{m}\right)$ операций. 
Иногда можно указать закономерности строения весовой функции Хемминга. Имеет место следующая теорема.

Теорема 12. Пусть $F(x) \in R[x]$ - реверсивный многочлен степени $m$, $m<n \leqslant T(F), \quad \mathcal{K}=L_{R}^{\overline{0, n-1}}(F)$ - линейный рекурсивный код. Если весовая функция Хемминга $W_{\mathcal{K}}(x, y)$ имеет вид

$$
W_{\mathcal{K}}(x, y)=x^{n}+\alpha x^{a} y^{n-a}+\beta x^{b} y^{n-b}+\gamma x^{c} y^{n-c},
$$

то справедливо равенство

$$
\left(\begin{array}{ccc}
1 & 1 & 1 \\
\delta_{a} & \delta_{b} & \delta_{c} \\
\delta_{a}^{2} & \delta_{b}^{2} & \delta_{c}^{2}
\end{array}\right) \cdot\left(\begin{array}{c}
\alpha \\
\beta \\
\gamma
\end{array}\right)=\left(\begin{array}{c}
2^{m}-1 \\
-\frac{1}{2} \\
\frac{2^{m-2}}{n}-\frac{1}{4}
\end{array}\right)
$$

гेe

$$
\delta_{a}=\frac{a}{n}-\frac{1}{2}, \quad \delta_{b}=\frac{b}{n}-\frac{1}{2}, \quad \delta_{c}=\frac{c}{n}-\frac{1}{2} .
$$

В частности, если (27) есть стандартная запись многочлена $W_{\mathcal{K}}(x, y)$, то этот многочлен полностью определяется значениями $a, b, c, m u n$.

Доказательство. Первое равенство системы (28) $\left(\alpha+\beta+\gamma=2^{m}-1\right)$ очевидно. Докажем второе равенство.

Если $F=f_{\mathbf{b}}, \mathbf{b} \in R^{m+1}$, то для любого вектора $\mathbf{a} \in R^{m+1}$ выполняется равенство $\delta\left(f_{\mathbf{a}}, u, t\right)=\delta\left(f_{\mathbf{a} \oplus \mathbf{b}}, u, t\right)$. Поэтому справедлива цепочка равенств

$$
\begin{aligned}
\sum_{\mathbf{a} \in R^{m+1}} \delta\left(f_{\mathbf{a}}, u, t\right) & =\delta\left(f_{\theta}, u, t\right)+\delta\left(f_{\mathbf{b}}, u, t\right)+\sum_{\mathbf{a} \neq \theta, \mathbf{a} \neq \mathbf{b}} \delta\left(f_{\mathbf{a}}, u, t\right)= \\
& =\sum_{\mathbf{a} \neq \theta, \mathbf{a} \neq \mathbf{b}, a_{m}=0} \delta\left(f_{\mathbf{a}}, u, t\right) .
\end{aligned}
$$

Пусть $\Delta^{\prime}=\left\{\delta\left(f_{\mathbf{a}}, u, t\right) \mid \mathbf{a} \neq \theta, \mathbf{a} \neq \mathbf{b}, a_{m}=0\right\}$. Согласно (26) и (27) набор чисел $\Delta^{\prime}$ состоит из

$\alpha$ чисел $\frac{a}{n}-\frac{1}{2}=\delta_{a}, \quad \beta$ чисел $\frac{b}{n}-\frac{1}{2}=\delta_{b} \quad$ и $\quad \gamma$ чисел $\frac{c}{n}-\frac{1}{2}=\delta_{c}$.

Ввиду реверсивности многочлена $F(x)$ на отрезке $(24)$ нет нулевых $(m+1)$ грамм. Поэтому согласно (11) имеем:

$$
0=\sum_{\mathbf{a} \in R^{m+1}} \delta\left(f_{\mathbf{a}}, u, t\right)=1+2 \sum_{\delta \in \Delta^{\prime}} \delta=1+2\left(\alpha \delta_{a}+\beta \delta_{b}+\gamma \delta_{c}\right) .
$$

Второе равенство системы (28) доказано. 
Докажем третье равенство. Аналогично предыдущим выкладкам

$$
\begin{aligned}
\sum_{\mathbf{a} \in R^{m+1}} \delta\left(f_{\mathbf{a}}, u, t\right)^{2} & =\delta\left(f_{\theta}, u, t\right)^{2}+\delta\left(f_{\mathbf{b}}, u, t\right)^{2}+2 \sum_{\delta \in \Delta^{\prime}} \delta^{2}= \\
& =\frac{1}{2}+2\left(\alpha \delta_{a}^{2}+\beta \delta_{b}^{2}+\gamma \delta_{c}^{2}\right)
\end{aligned}
$$

Отсюда и из (10) следует третье равенство системы (28).

Докажем второе утверждение теоремы. Определитель матрицы системы (28) есть определитель Ван-дер-Монда (см., например, [2]). Если выражение (27) есть стандартная запись многочлена $W_{\mathcal{K}}(x, y)$ и числа $\delta_{a}, \delta_{b}, \delta_{c}$ попарно различны, то матрица системы (28) обратима, и вектор $(\alpha, \beta, \gamma)$ однозначно определяется значениями $a, b, c, m$ и $n$.

Функция $W_{\mathcal{K}}(x, y)$ имеет вид (27), например когда $F(x)$ - неприводимый многочлен,

$$
n=T(F)=\frac{2^{m}-1}{d}
$$

и $d \mid 2^{j}+1$ для некоторого $j \geqslant 1$ (см. [9]).

Основными недостатками метода БПФ являются высокая трудоемкость (нелинейный ранг отрезка может быть велик) и необходимость вычислять все ВХМ для того, чтобы найти хотя бы один из них. В следующем разделе будет описан метод, позволяющий находить один ВХМ, не находя при этом все множество ВХМ.

\section{4. Алгоритм, использующий ортогонализацию}

Пусть $u \in R^{\infty}$-ЛРП с реверсивным минимальным многочленом $F(x) \in R[x]$, $\operatorname{deg} F(x)=m, F(1)=0, m<n \leqslant T(F), t=m+n-1$. В этом разделе предложен алгоритм поиска одного ВХМ отрезка (1) степени не выше $(m-1)$ с трудоемкостью $O\left(m n^{3}\right)$. Для «достаточно коротких» отрезков (1) этот метод эффективнее изложенного в предыдущих разделах метода БПФ.

Будем говорить, что линейная функция $(\mathbf{a}, \mathbf{x}), \mathbf{a} \in R^{m}$, равновероятна на множестве $X \subset R^{m}$, если

$$
|\{\mathbf{x} \in X:(\mathbf{a}, \mathbf{x})=0\}|=\frac{1}{2}|X|
$$

В основе предлагаемого метода поиска ВХМ лежат алгоритм ортогонализации Грама-Шмидта (см., например, [2]) и следующая лемма. 
Лемма 13. Если $X \subset R^{m}$ и $X \neq R^{m}$, то сущзествует такой вектор $\mathbf{a} \in R^{m} \backslash\{\theta\}$, что функция $(\mathbf{a}, \mathbf{x})$ не равновероятна на множестве $X$.

Доказательство. Сопоставим множеству $X$ векторное пространство $L_{X}$ над $\mathbb{R}$, состоящее из всех функций типа $X \rightarrow \mathbb{R}$. Введем на пространстве $L_{X}$ скалярное произведение $S_{X}$ :

$$
S_{X}(f, g)=\frac{1}{|X|} \sum_{\mathbf{x} \in X} f(\mathbf{x}) g(\mathbf{x}) \quad \forall f, g \in L_{X}
$$

(сумма здесь - в поле $\mathbb{R}$ ), и построим тем самым евклидово пространство $\mathcal{L}_{X}=\left(L_{X}, S_{X}\right)$ над полем $\mathbb{R}$. Очевидно, $\operatorname{dim} \mathcal{L}_{X}=|X|$.

Пусть $\chi_{\mathbf{a}}(\mathbf{x})$ - характер элементарной абелевой 2-группы $\left(\mathbb{Z}_{2}^{m},+\right)$ :

$$
\forall \mathbf{a}, \mathbf{x} \in R^{m}: \quad \chi_{\mathbf{a}}(\mathbf{x})=(-1)^{(\mathbf{a}, \mathbf{x})},
$$

a $\varphi_{\mathbf{a}}=\left.\chi_{\mathbf{a}}\right|_{X}$ - ограничение характера $\chi_{\mathbf{a}}$ на множество $X, \mathbf{a} \in R^{m}$. Тогда $\varphi_{\mathbf{a}} \in L_{X}$ для всякого $\mathbf{a} \in R^{m}$. Предположим теперь, что для всякого $\mathbf{a} \in R^{m} \backslash\{\theta\}$ функция $(\mathbf{a}, \mathbf{x})$ равновероятна на множестве $X$. Это означает, что

$$
\forall \mathbf{a} \in R^{m}: \varphi_{\mathbf{a}} \not \equiv 0, \quad \forall \mathbf{a}, \mathbf{b} \in R^{m}:(\mathbf{a} \neq \mathbf{b}) \Rightarrow\left(S_{X}\left(\varphi_{\mathbf{a}}, \varphi_{\mathbf{b}}\right)=0\right)
$$

Таким образом, $\left\{\varphi_{\mathbf{a}} \mid \mathbf{a} \in R^{m}\right\}-$ ортогональная и, следовательно, линейная независимая система векторов из $\mathcal{L}_{X}$, состоящая из $2^{m}$ векторов (в частности, $\varphi_{\mathbf{a}} \neq \varphi_{\mathbf{b}}$ при $\left.\mathbf{a} \neq \mathbf{b}\right)$. Отсюда $|X|=\operatorname{dim} \mathcal{L}_{X} \geqslant 2^{m}$, а так как $X \subset R^{m}$, то $X=R^{m}$. Противоречие.

Рассмотрим теперь отрезок (1)

$$
u(0), u(1), \ldots, u(t-1)
$$

и семейство $m$-грамм (5). По условию $n \leqslant T(F)=T(u)$, поэтому $t<T(u)+m$, и условие (6) выполнено. Это означает, что в семействе (5) все $m$-граммы попарно различны (см. доказательство утверждения 3 ), и семейство (5) есть множество. Обозначим множество (5) через $X$. Очевидно, $|X|=\operatorname{dim} \mathcal{L}_{X}=n$. 
Упорядочим некоторым образом множество $R^{m}$ :

$$
\mathbf{a}_{0}, \mathbf{a}_{1}, \ldots, \mathbf{a}_{2^{m}-1}
$$

и положим $f_{i}=\varphi_{\mathbf{a}_{i}}, \quad i=0,1, \ldots, 2^{m}-1$. Рассмотрим последовательность векторов

$$
f_{0}, f_{1}, \ldots, f_{i}, \ldots
$$

и, применив к ней стандартный процесс ортогонализации (см., например, [2]), найдем такое минимальное $i$, что вектор $f_{i}$ не ортогонален линейной оболочке

$$
\left(f_{0}, f_{1}, \ldots, f_{i-1}\right)_{\mathbb{R}}
$$

Поскольку $\operatorname{dim} \mathcal{L}_{X}=n$, такое $i$ не превосходит $n \leqslant 2^{m}-1$.

Пусть вектор $f_{i}$ не ортогонален линейной оболочке (29). Это означает, что существует такое $j \in\{0,1, \ldots, i-1\}$, что вектор $f_{i}$ не ортогонален вектору $f_{j}$, т. е. функция $\left(\mathbf{a}_{i} \oplus \mathbf{a}_{j}, \mathbf{x}\right)$ не равновероятна на множестве $X$ : иными словами,

$$
\delta\left(f_{\mathbf{a}_{i} \oplus \mathbf{a}_{j}}, u, t\right) \neq 0 .
$$

Если $\delta\left(f_{\mathbf{a}_{i} \oplus \mathbf{a}_{j}}, u, t\right)>0$, то $f_{\mathbf{a}_{i} \oplus \mathbf{a}_{j}}(\mathbf{x})-$ ВХМ отрезка (1) со свойством

$$
\operatorname{deg} f_{\mathbf{a}_{i} \oplus \mathbf{a}_{j}}(\mathbf{x}) \leqslant m-1 .
$$

Пусть $\delta\left(f_{\mathbf{a}_{i} \oplus \mathbf{a}_{j}}, u, t\right)<0$. Зная отрезок (1), можно построить отрезок (25) рекурренты

$$
v=f_{\mathbf{a}_{i} \oplus \mathbf{a}_{j}} \cdot u \in L_{R}(F) .
$$

Поскольку $\delta\left(f_{\mathbf{a}_{i} \oplus \mathbf{a}_{j}}, u, t\right)<0$, доля нулей на отрезке (25) меньше $1 / 2$. Так как $F(1)=0$, в семействе $L_{R}(F)$ лежит ЛРП $(1,1, \ldots, 1, \ldots)$. Тогда $\bar{v}=$ $v \oplus(1,1, \ldots, 1, \ldots)$ также принадлежит семейству $L_{R}(F)$, и на отрезке

$$
\bar{v}(0), \bar{v}(1), \ldots, \bar{v}(n-1)
$$

доля нулей больше $1 / 2$. Согласно [2] существует многочлен $f_{\mathbf{b}}, \mathbf{b} \in R^{m}$, со свойством $\bar{v}=f_{\mathbf{b}} \cdot u$. Тогда $f_{\mathbf{b}}-$ ВХМ отрезка (1) и $\operatorname{deg} f_{\mathbf{b}} \leqslant m-1$.

Оценим трудоемкость описанного выше алгоритма вычисления ВХМ. Для того чтобы найти вектор $f_{i}$, не ортогональный линейной оболочке (29), необходимо осуществить не более $n=|X|$ шагов алгоритма ортогонализации (см. [2]). На $s$-м, $1 \leqslant s \leqslant n$, шаге алгоритма ортогонализации необходимо вычислить $(s-1)$ дробей, в числителе и знаменателе которых стоят скалярные произведения $S_{X}(\cdot, \cdot)$. Вычисление одного такого скалярного произведения требует $O(m n)$ операций в поле $\mathbb{Q}$. Поэтому вектор $f_{i}$ вычисляется за $O\left(m n^{3}\right)$ операций в поле $\mathbb{Q}$. Одновременно вычисляется вектор $f_{j}$, которому не ортогонален $f_{i}$. 
Вычисления закончены, если $\delta\left(f_{\mathbf{a}_{i} \oplus \mathbf{a}_{j}}, u, t\right)>0$. Если же $\delta\left(f_{\mathbf{a}_{i} \oplus \mathbf{a}_{j}}, u, t\right)<0$, то нужно за $m n=O\left(n^{2}\right)$ операций вычислить отрезок (25) и за $O(n)$ операций инвертировать его. Вычисление многочлена $f_{\mathrm{b}}$ связано с обращением матрицы

$$
\left(\begin{array}{ccccc}
u(0) & u(1) & u(2) & \ldots & u(m-1) \\
u(1) & u(2) & u(3) & \ldots & u(m) \\
u(2) & u(3) & u(4) & \ldots & u(m+1) \\
\vdots & \vdots & \vdots & \ddots & \vdots \\
u(m-1) & u(m) & u(m+1) & \ldots & u(2 m-2)
\end{array}\right)
$$

которое может быть осуществлено за $O\left(\mathrm{~m}^{3}\right)$ операций в $R$. Таким образом, для нахождения ВХM $f_{\mathbf{b}}$ требуется $O\left(m n^{3}\right)$ операций в $R$.

Суммируя сказанное, получаем, что справедлива следующая теорема.

Теорема 14. Пусть $u \in R^{\infty}-$ ЛРП с реверсивным минимальным многочленом $F(x) \in R[x], \operatorname{deg} F(x)=m, F(1)=0, m<n \leqslant T(F), t=m+n-1$. Тогда существует алгоритм, вычисляюший ВХМ отрезка (1) степени не выше $m-1$ с трудоемкостью $O\left(m n^{3}\right)$ операций в полях $\mathbb{Q} u R$.

Если наложить на характеристический многочлен $F(x)$ дополнительные ограничения, то поиск ВХМ можно существенно ускорить. Об этом - следующий раздел.

\section{5. Поиск ВХМ: сепарабельный случай}

Пусть $F(x) \in R[x]-$ реверсивный сепарабельный многочлен, т. е. $F(x)-$ реверсивный многочлен, не имеющий кратных корней в своем поле разложения. Рассмотрим его каноническое разложение над $R$ :

$$
F(x)=G_{1}(x) G_{2}(x) \ldots G_{k}(x),
$$

где $G_{i}(x)$ - неприводимый над $R$ многочлен степени $m_{i}, i=1,2, \ldots, k$. Обозначим через $m$ наименьшее общее кратное чисел $m_{1}, m_{2}, \ldots, m_{k}$ :

$$
m=\left[m_{1}, m_{2}, \ldots, m_{k}\right] .
$$

Тогда $S=G F\left(2^{m}\right)$ - общее поле разложения многочленов $G_{1}, G_{2}, \ldots, G_{k}$ и

$$
T(F) \mid \tau=2^{m}-1 .
$$

Рассмотрим ЛРП $u \in R^{\infty}$ с минимальным многочленом $F(x)$. Построим рекурренту $v \in R^{\infty}$ со свойствами $T(v) \mid \tau, \operatorname{rang} v \leqslant m$ и

$$
|\{i \in\{0,1, \ldots, \tau-1\}: u(i)=v(i)\}| \geqslant 2^{m-1} .
$$

Соотношение (31), означающее, что последовательность $v$ аппроксимирует последовательность $u$, будем в дальнейшем записывать в виде $u \approx v$. 
Обозначим через $\operatorname{tr}$ функцию «след» из поля $S$ в поле $R$ :

$$
\operatorname{tr}(x)=x+x^{2}+x^{2^{2}}+\cdots+x^{2^{m-1}} .
$$

Пусть $\vartheta \in S-$ примитивный элемент: $\langle\vartheta\rangle=S^{*}$. Рассмотрим циклический базис $\vec{\vartheta}=\left(e, \vartheta, \vartheta^{2}, \ldots, \vartheta^{m-1}\right)$ пространства $R_{R}$ и базис $\vec{\varepsilon}=\left(\varepsilon_{0}, \varepsilon_{1}, \ldots, \varepsilon_{m-1}\right)$, двойственный к циклическому (см., например, [2]):

$$
\operatorname{tr}\left(\varepsilon_{i} \cdot \vartheta^{j}\right)=\delta_{i, j},
$$

где $\delta_{i, j}-$ символ Кронекера, $0 \leqslant i, j \leqslant m-1$. Тогда для всякого элемента $a \in S$ верно равенство

$$
\forall x \in S: \quad \operatorname{tr}(a x)=\left(a_{\vec{\vartheta}}, x_{\vec{\varepsilon}}\right),
$$

где $a_{\vec{\vartheta}} \in R^{m}$ - строка координат вектора $a$ в базисе $\vec{\vartheta}, x_{\vec{\varepsilon}} \in R^{m}-$ строка координат вектора $x$ в базисе $\vec{\varepsilon}$.

Пусть $\vartheta^{\delta_{s}}$ - корень многочлена $G_{s}(x)$ в поле $S, 1 \leqslant \delta_{s} \leqslant \tau-1, s=1,2, \ldots, k$. Тогда для всякого $i \geqslant 0$ верна цепочка равенств

$$
u(i)=\sum_{s=1}^{k} \operatorname{tr}\left(a_{s} \vartheta^{i \delta_{s}}\right)=\operatorname{tr}\left(\Phi\left(\vartheta^{i}\right)\right),
$$

где $\Phi(x)=a_{1} x^{\delta_{1}}+a_{2} x^{\delta_{2}}+\cdots+a_{k} x^{\delta_{k}}$, и все коэффициенты $a_{s} \in S$ отличны от нуля. Рассмотрим булеву функцию $f: R^{m} \rightarrow R$, определенную равенством

$$
\forall \mathbf{x} \in R^{m}: \quad f(\mathbf{x})=\operatorname{tr}\left(\Phi\left(\mathbf{x} \cdot \vec{\varepsilon}^{T}\right)\right),
$$

где ${ }^{T}{ }^{T} »-$ операция транспонирования.

Рассмотрим минимальный многочлен $G(x) \in R[x]$ элемента $\vartheta \in S$ и $w \in L_{R}(G)$ - рекурренту с начальным вектором $e_{\vec{\varepsilon}}$. Тогда, как легко видеть,

$$
\forall i \geqslant 0: \quad w(i)=\operatorname{tr}\left(\vartheta^{i}\right) \quad \text { и } \quad \vartheta^{i}=\sum_{s=0}^{m-1} \varepsilon_{s} w(i+s) .
$$

Если $\mathbf{x} \in R^{m} \backslash\{\theta\}$, то $\mathbf{x}=(w(i), w(i+1), \ldots, w(i+m-1))$ для некоторого $i \geqslant 0$. Ввиду (32) - (35) равенство

$$
f(\mathbf{x})=(\mathbf{a}, \mathbf{x})
$$

эквивалентно соотношению $u(i)=\operatorname{tr}\left(a \vartheta^{i}\right)$, где $a_{\vec{\vartheta}}=\mathbf{a}$. Поэтому если $v_{\mathbf{a}} \in L_{R}(G)$ - рекуррента с начальным вектором $a_{\vec{\varepsilon}}$, то равенство (36) эквивалентно соотношению

$$
u(i)=v_{\mathbf{a}}(i)
$$


Эквивалентность соотношений (36) и (37) означает, что поиск рекуррент $v \in L_{R}(G)$ со свойством $u \approx v$ эквивалентен поиску линейных статистических аналогов функции $f(\mathbf{x})$ (см., например, [1]). Действительно, справедливо следующее утверждение.

Утверждение 15. Если $v_{\theta}=0, u$

$$
\forall \mathbf{a} \in R^{m}: \quad \delta_{\mathbf{a}}(u)=\frac{1}{\tau}\left|\left\{i \in\{0,1, \ldots, \tau-1\}: u(i)=v_{\mathbf{a}}(i)\right\}\right|-\frac{1}{2},
$$

то верно равенство

$$
\forall \mathbf{a} \in R^{m}: \quad \delta_{\mathbf{a}}(u)=\frac{1}{\tau}\left(\Delta_{\mathbf{a}}(f)-\frac{1}{2}\right) .
$$

Доказательство. Ввиду эквивалентности соотношений (36) и (37) имеем:

$$
\begin{aligned}
\delta_{\mathbf{a}}(u) & =\frac{1}{\tau}\left|\left\{i \in\{0,1, \ldots, \tau-1\}: u(i)=v_{\mathbf{a}}(i)\right\}\right|-\frac{1}{2}= \\
& =\frac{1}{\tau}\left(\left|\left\{\mathbf{x} \in R^{m}: f(\mathbf{x})=(\mathbf{a}, \mathbf{x})\right\}\right|-1\right)-\frac{1}{2}= \\
& =\frac{2^{m-1}-1}{2^{m}-1}+\frac{1}{\tau} \Delta_{\mathbf{a}}(f)-\frac{1}{2}=\frac{1}{\tau} \Delta_{\mathbf{a}}(f)-\frac{1}{2 \tau}= \\
& =\frac{1}{\tau}\left(\Delta_{\mathbf{a}}(f)-\frac{1}{2}\right) .
\end{aligned}
$$

Поскольку

$$
\sum_{\mathbf{a} \in R^{m}} \Delta_{\mathbf{a}}(f)=(-1)^{f(\theta)} 2^{m-1}=2^{m-1}
$$

среди коэффициентов $\Delta_{\mathbf{a}}(f), \mathbf{a} \in R^{m}$, есть положительные. Так как коэффициенты $\Delta_{\mathbf{a}}(f), \mathbf{a} \in R^{m}$, - целые, по утверждению 15 существует такой вектор $\mathbf{a} \in R^{m}$, что $\delta_{\mathbf{a}}(u)>0$. Тогда $u \approx v_{\mathbf{a}}$. Суммируя сказанное, получим следующий алгоритм вычисления ЛРП $v_{\mathbf{a}} \in L_{R}(G)$, аппроксимирующей последовательность $u$. Предполагается, что до начала работы алгоритма в памяти создана таблица значений функции $\operatorname{tr}(\cdot)$.

Алгоритм 16. (вычисление рекурренты $v \in L_{R}(G)$, аппроксимирующей $u$ )

Вход: ЛРП $u \in L_{R}(F)$, значение $m$, примитивный элемент $\vartheta$ поля $S=$ $G F\left(2^{m}\right), G(x)$ - минимальный многочлен элемента $\vartheta$.

Выход: ЛРП $v \in L_{R}(G)$ со свойством $u \approx v$. 
Шаг 1. По адресу $\theta \in R^{m}$ записать $f(\theta)=0$. Положить $i=0$.

Шаг 2. Положить $\mathbf{x}=\left(\operatorname{tr}(e), \operatorname{tr}(\vartheta), \ldots, \operatorname{tr}\left(\vartheta^{m-1}\right)\right)$.

Шаг 3. По адресу $\mathbf{x}$ записать $f(\mathbf{x})=u(i)$.

Шаг 4. Если $i=\tau-1$, то перейти на шаг 6.

Шаг 5. Присвоить х значение $\mathbf{x} S(G)$, где $S(G)$ - сопровождающая матрица многочлена $G(x)$, увеличить $i$ на 1 и перейти на шаг 3 .

Шаг 6. По построенной таблице функции $f$ методом БПФ найти набор коэффициентов статистической структуры $\Delta_{\mathbf{a}}(f), \mathbf{a} \in R^{m}$.

Шаг 7. Перебирая все векторы $\mathbf{a} \in R^{m}$ в порядке двоичного счета, найти первый по счету вектор а, для которого $\Delta_{\mathbf{a}}(f) \geqslant 1$.

Шаг 8. Найти элемент $a \in S$, для которого $a_{\vec{\vartheta}}=\mathbf{a}$.

Шаг 9. Вычислить $a_{\vec{\varepsilon}}$. ЛРП $v \in L_{R}(G)$ с начальным вектором $a_{\vec{\varepsilon}}-$ искомая.

Теорема 17. Пусть $F(x) \in R[x]-$ реверсивный сепарабельный многочлен с каноническим разложением (30), $\operatorname{deg} G_{i}(x)=m_{i}, i=1,2, \ldots, k, m=\left[m_{1}, \ldots, m_{k}\right]$, $S=G F\left(2^{m}\right),\langle\vartheta\rangle=S^{*}, G(x) \in R[x]-$ минимальный многочлен элемента $\vartheta, u \in L_{R}(F)$ - рекуррента с минимальным многочленом $F(x)$. Тогда алгоритм 16 строит такую ЛРП $v \in L_{R}(G)$, что $u \approx v$, с трудоемкостью $O\left(m 2^{m}\right)$ операций в полях $\mathbb{Q}$ и $R$.

Доказательство. Корректность работы алгоритма 16 уже доказана. Вычислим его трудоемкость.

Домножение на сопровождающую матрицу $S(G)$ осуществляется за $O(m)$ операций в $R$. Поэтому таблица функции $f$ строится за $O\left(m 2^{m}\right)$ операций в $R$. Шаг 6 осуществляется за $O\left(m 2^{m}\right)$ операций. Перебор на шаге 7 осуществляется за $O\left(2^{m}\right)$ операций. Шаг 8 требует $O\left(m^{3}\right)$ операций в $R$. Вычисление вектора $a_{\vec{\varepsilon}}$ осуществляется за $O\left(\mathrm{~m}^{3}\right)$ операций в $R$. Суммарная трудоемкость алгоритма 16 составляет $O\left(m 2^{m}\right)$ операций в $R$.

Отметим, что в предшествующих работах теорема 17 была доказана в ситуации, когда $F(x)$ - неприводимый над $R$ многочлен. В теореме 17 рассматривается более общий случай: многочлен $F(x)$ сепарабелен, т. е. равен произведению попарно разных неприводимых над $R$ многочленов. 
Следствие 18. Если в условиях теоремы $17 \quad F(1)=F(\vartheta)=0 u \operatorname{deg} F(x)=$ $\mu$, то у отрезка

$$
u(0), u(1), \ldots, u(\tau+\mu-2)
$$

существует $\mathrm{BXM} \varphi(x) \in R[x]$ со свойствали $\operatorname{deg} \varphi(x)<\mu u$

$$
\delta(\varphi, u, \tau+\mu-1) \geqslant \frac{2^{\frac{m}{2}}-1}{2^{m+1}-2} .
$$

ВХМ $\varphi$ может быть построен за $O\left(\mu^{3}+m 2^{m}\right)$ операций в полях $\mathbb{Q} u R$.

Доказательство. Условие $F(\vartheta)=0$ означает, что $G(x) \mid F(x)$ и, следовательно, $L_{R}(G) \subseteq L_{R}(F)$. Поэтому все ЛРП $v_{\mathbf{a}}$, $\mathbf{a} \in R^{m}$, принадлежат $L_{R}(F)$. Из равенства Парсеваля следует, что

$$
\max _{\mathbf{a} \in R^{m}}\left|\Delta_{\mathbf{a}}(f)\right| \geqslant 2^{\frac{m}{2}-1} .
$$

Ввиду (38) и очевидного неравенства $|x-y| \geqslant|x|-|y|$ имеем:

$$
\forall \mathbf{a} \in R^{m}: \quad\left|\delta_{\mathbf{a}}(u)\right| \geqslant \frac{1}{\tau}\left(\left|\Delta_{\mathbf{a}}(f)\right|-\frac{1}{2}\right),
$$

откуда

$$
\max _{\mathbf{a} \in R^{m}}\left|\delta_{\mathbf{a}}(u)\right| \geqslant \frac{2^{\frac{m}{2}}-1}{2^{m+1}-2} .
$$

Рассмотрим вектор $\mathbf{c} \in R^{m}$, для которого

$$
\left|\delta_{\mathbf{c}}(u)\right|=\max _{\mathbf{a} \in R^{m}}\left|\delta_{\mathbf{a}}(u)\right| .
$$

Если $\delta_{\mathbf{c}}(u)>0$, то $u \approx v_{\mathbf{c}}$. Поскольку $F(x)$ - минимальный многочлен ЛРП $u$,

$$
v_{\mathbf{c}}=\psi(x) \cdot u
$$

для некоторого многочлена $\psi(x) \in R[x]$ со свойством $\operatorname{deg} \psi(x)<\operatorname{deg} F(x)$. Тогда $(1 \oplus \psi(x)) \cdot u \approx 0$, и для многочлена $\varphi(x)=1 \oplus \psi(x)$ получаем:

$$
\delta(\varphi, u, \tau+\mu-1)=\delta_{\mathbf{c}}(u) \geqslant \frac{2^{\frac{m}{2}}-1}{2^{m+1}-2} .
$$


Пусть $\delta_{\mathbf{c}}(u)<0$. Поскольку $F(1)=0$, рекуррента $\bar{v}_{\mathbf{c}}=v_{\mathbf{c}} \oplus(1,1,1, \ldots)$ принадлежит семейству $L_{R}(F)$ и $u \approx \bar{v}_{\mathbf{c}}$. Тогда

$$
\bar{v}_{\mathbf{c}}=\psi_{1}(x) \cdot u
$$

для некоторого многочлена $\psi_{1}(x) \in R[x]$ со свойством $\operatorname{deg} \psi_{1}(x)<\operatorname{deg} F(x)$, и $\left(1 \oplus \psi_{1}(x)\right) \cdot u \approx 0$. Для многочлена $\varphi(x)=1 \oplus \psi_{1}(x)$ имеем:

$$
\delta(\varphi, u, \tau+\mu-1)=\left|\delta_{\mathbf{c}}(u)\right| \geqslant \frac{2^{m / 2}-1}{2^{m+1}-2} .
$$

Вычислим трудоемкость нахождения многочлена $\varphi(x)$. Набор чисел $\delta_{\mathbf{a}}(u)$, $\mathbf{a} \in R^{m}$, вычисляется за $O\left(m 2^{m}\right)$ операций в $\mathbb{Q}$. Поиск вектора $\mathbf{c}$, для которого выполняется равенство (39), осуществляется за $O\left(2^{m}\right)$ операций сравнения рациональных чисел. Вычисление многочленов $\psi$ и $\psi_{1}$ по формулам (40) и (41) требует $O\left(\mu^{3}\right)$ операций в $R$. Таким образом, суммарная трудоемкость вычисления многочлена $\varphi(x)$ составляет $O\left(\mu^{3}+m 2^{m}\right)$ операций в полях $\mathbb{Q}$ и $R$.

\section{6. Заключение}

Результаты работы позволяют сделать следующие выводы:

1. Задача вычисления ВХМ отрезка (1), а также их преобладаний может быть решена за $O\left(m 2^{m}\right)$ операций в поле $\mathbb{Q}$, где $m-$ нелинейный ранг отрезка (1). При «больших», т. е. близких к периоду последовательности $u$, значениях $t$ алгоритм БПФ позволяет существенно понизить трудоемкость по сравнению с тотальным перебором.

2. Метод БПФ может быть применен и к задаче вычисления весовой функции Хемминга линейного рекурсивного кода.

3. В случае «короткого» отрезка (1) алгоритм БПФ малоэффективен. В этой ситуации целесообразнее использовать алгоритм из раздела 4 , трудоемкость которого полиномиальна относительно $t$. Вместе с тем, работая с короткими отрезками (1), можно приобрести «лишние» ВЛС, отражающие специфику самого отрезка, а не устройства, его вырабатывающего.

4. Пусть $2^{m}-$ порядок минимального поля разложения минимального многочлена $F(x)$ рекурренты $u$. Факт сепарабельности многочлена $F(x)$ существенно упрощает задачу поиска одного ВХМ отрезка

$$
u(0), u(1), \ldots, u(\tau+\mu-2),
$$

где $\tau=2^{m}-1$ и $\mu=\operatorname{deg} F(x)$. 
Автор признателен А.В. Анашкину за постановку задачи, О.В.Камловскому и О.В. Денисову за обсуждение полученных результатов, В. О. Дрелихову за существенные замечания по тексту статьи.

\section{Список литературы}

[1] Бабаш А. В., Шанкин Г. П., Криптография, М. : Солон-Р, 2002, 512 с.

[2] Глухов М.М., Елизаров В.П., Нечаев А.А., Алгебра. В 2 m., М. : Гелиос-АРВ, 2003, $336+415 \mathrm{c}$.

[3] Глухов М.М., Козлитин О.А., Шапошников В.А., Шишков А.Б., Задачи и упражнения по математической логике, дискретным функциям и теории алгоритмов, СПб. : Лань, 2008, 112 c.

[4] Глухов М. М., Шишков А. Б., Математическая логика. Дискретные функиии. Теория алгоритмов, СПб. : Лань, 2012, 416 с.

[5] Гонсалес С., Коусело Е., Марков В., Нечаев А., "Параметры рекурсивных МДР-кодов", Дискретная математика, $12: 4$ (2000), 3-24.

[6] Лидл Р., Нидеррайтер Г., Конечные поля. В 2 m., М. : Мир, 1988, 808 с.

[7] Логачев О.А., Сальников А.А., Смышляев С.В., Ященко В.В., Булевы функции в теории кодирования и криптологии, М.: МЦНМО, 2012, 584 с.

[8] Мак-Вильямс Ф. Д., Слоэн Н. Д. А., Теория кодов, исправляющих ошибки, М. : Связь, 1979, $744 \mathrm{c}$.

[9] McEliece R. J., "Irreducible cyclic codes and Gauss sums". In: "Combinatorics", DordrechtBoston: Reidel, 1975, 185-202. 